\title{
A Practical Approach to the Symbolic Debugging of Parallelized Code ${ }^{1}$
}

\author{
Patricia Pineo \\ ppineo@alleg.edu \\ (814) 332-2883
}

\author{
Mary Lou Soffa \\ soffa@cs.pitt.edu \\ (412) 624-8425
}

Computer Science Department

University of Pittsburgh

Pittsburgh, PA 15260

Fax: (412) 624-5299

\begin{abstract}
A practical technique is presented that supports the debugging of parallelized code through global renaming and name reclamation. Global renaming creates single assignment code for programs destined to be parallelized. After parallelization, a reclamation of names not useful for either the execution or debugging of the code is performed. During execution non-current values can then be tracked and reported to the debugger. Results of experimentation indicate the enlargement of the name space is reasonable and that virtually all non-current values are reportable. The technique is independent of the transformations chosen to parallelize the code.
\end{abstract}

\section{Introduction}

The importance of renaming as a program transformation is growing with the increased recognition of its value in program analysis |cypres7,wortse9. The two forms of renaming that have emerged as particularly useful are single assignment and static single assignment. In single assignment each assignment is made into a unique variable, and once computed, a variable will never be altered. Static single assignment differs in that although only one assignment statement may appear in the code for each variable, that statement can be repeatedly executed (as in a loop).

The usefulness of static single assignment has been demonstrated as a pre-processing stage to simplify dataflow analysis during the application of optimizing transformations ${ }^{\text {[PrRwzol] }}$. It has also been shown useful in applying optimizations such as induction variable elimination ${ }^{[\text {Wolsg2] }}$. Under the assumption of single assignment code, the problem of partitioning sequential code for a parallel environment is "drastically simplified" [BANaRosg] . Single assignment is also shown useful in register allocation optimizations ${ }^{\text {[Boda91] }}$.

\footnotetext{
' Partially supported by National Science Foundation Grant CCR-91090809 to the University of Pittsburgh.

- Presenting Author
} 
Although these single assignment forms have been shown to be useful for program analysis, their use during program execution has been restricted due to the impracticality of storage enlargement. In this paper we develop a technique that enables the use of renamed code during program execution. This is made possible by selectively reclaiming names prior to code execution.

This technique developed is another application of single assignment code that of symbolic debugging of code that has been transformed by either traditional optimizations or parallelizing transformations. Because of code modification, deletion, reorganization and parallelization, the actual values of variables seen at breakpoints during runtime will often be different from the values expected by the programmer viewing the sequential, untransformed code. One approach to the problem of non-current variables is to force the programmer to directly view and debug the transformed code, but this approach requires that the user have familiarity with the parallel constructs available, the architecture and the mapping from the source to transformed code. A preferable approach is to allow the user to execute the transformed code on the parallel system but to debug the code from the viewpoint of the sequential code.

This approach to the problem of debugging transformed code has been

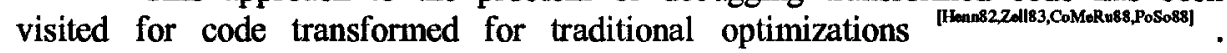
These techniques all create a history of specific optimizations performed with the objective of unwinding the optimizations selectively during debugging in order to recover non-current variables. These techniques work with a subset of 3-4 specific optimizations and must be expanded if other optimizations are applied. They are more successful when optimizations are local, becoming complex and expensive when code is moved across basic blocks. The present work differs in that expansive code motion does not increase the complexity, the work is not transformation dependent and the code is not modified during debugging. This last point is significant because code that is modified for debugging may execute during debugging runs, and then fail when debugging is not invoked.

This problem has also been considered by Gupta $^{[0 \mathrm{pppt83]}}$ in relation to debugging code reorganized by a trace scheduler. Gupta's technique enables expected values in reordered VLIW code to be reported. It requires debugging requests to be made in advance, and the recompilation of selected traces. The present work differs in that it allows inspection of all variables at any breakpoint without recompilation, and it is not architecture specific.

Each of these methods employs ad hoc techniques for saving and recovering non-current values in newly defined storage locations. By contrast, Global Renaming allows values to be stored and recovered in a unified way, without consideration of any code transformation. Because each value is carried in a unique name, renamed code can be transformed by unrestricted parallelizing transformations, and still be successfully debugged.

Unlike using renaming as a purely analytical technique, renaming in debugging has a problem in the explosion of the storage associated with single assignment programs. This problem is resolved in this work by the application of a second stage that reclaims names not needed for either parallel execution or debugging before execution occurs. 
Thus, this paper presents a practical approach to the use of renaming in debugging of parallelized code. The techniques have been implemented and experimental results are presented. Through these experimental results, we demonstrate that after name reclamation, the storage expansion caused by the renaming is reasonable and virtually all non-current names can be reported.

There are several additional advantages of using the renaming approach for debugging transformed code. First, the renaming allows the exploitation of additional parallelism in program code by reducing data dependencies. Further, this same analysis can be used to simplify the application of several standard parallelizing transformations. Finally the technique imposes no restrictions on the number or type of parallelizing transformations applied. This allows the approach to interface easily with a variety of transformational packages aimed at diverse target architectures.

In this extended summary, we first present an overview of the technique. We then present the two analysis techniques, focusing on the reclamation of names. Experimental results are presented, showing that this approach is indeed a practical approach.

\section{Overview of Debugging with Global Renaming}

Practical high-level debugging with global renaming is accomplished in five stages. An overview of our technique is given in the algorithm of Figure 1. Two stages (numbered one and three) are introduced to bracket the application of parallelizing transformations. The primary purpose of the first stage is the renaming of the code and the production of AVAIL sets, which are sets that retain the current names of variables that should be reportable after the execution of the associated statement number in the original program. These sets provide the value tracking capability used by the debugger at execution time.

Algorithm -- High-level debugging of parallelized code

1. Globally rename code (IN: original code, OUT: single assignment code, AVAIL sets)

2. Apply user chosen parallelization transformations(IN: SA code, OUT:parallelized code)

3. Reclain unneeded names (IN: parallelized SA code,

OUT: reduced name parallelized code, INOUT: AVAIL)

4. Compile (IN: reduced name parallelized code, OUT: executable code)

5. Execute code through debugger modified to access AVAlL sets when values ane requested

Figure 1 - Overview of the debugging technique

A simple program is shown passing through the stages of the system in Figure 2. Initially the code is globally renamed. This first stage produces a semantically equivalent version of the program in single assignment form, which assigns each (potentially non-current) value a unique storage name. The current names at each statement are retained in the AVAIL sets. The reduction of undesirable data dependencies by the renaming can also be observed in the example. Antidependencies (e.g., statement S1 $\delta^{-1} \mathrm{~S} 3$ ), and output dependencies (S6 $\mathrm{d}^{\circ} \mathrm{S} 7$ ) are removed in the renamed code. The resulting code has been freed 


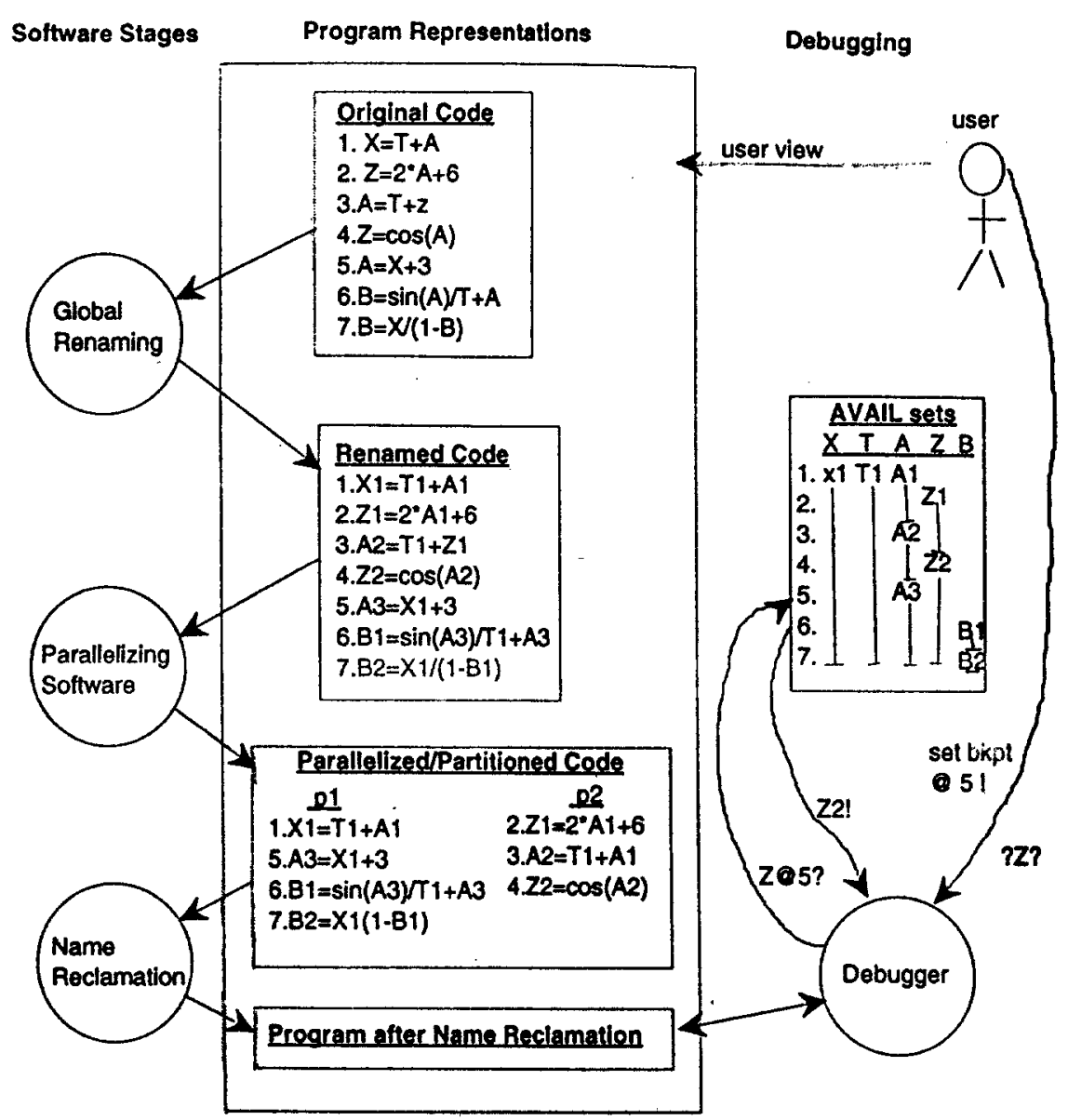

Figure 2 - Debugging with Global Renaming

from about half of the original data dependencies and thus allows a more aggressive exploitation of parallelism.

The single assignment code can now be parallelized by software targeted for any desired architecture. The choice of transformations applied in this process are not important to the debugging system. Regardless of where variables are moved, their version names carry the tag required by the debugger for later inquiries.

Once the parallelized code has been finalized, it may be that not all the names introduced through renaming are necessary. Some variables must be retained because they enable the reporting of a non-current value at debug time. In this example, the programmer (debugging from the viewpoint of the sequential code) may insert a breakpoint after statement 5 and request the value of $Z$. This breakpoint maps to statement of the parallelized code and the associated AVAIL set indicates that $\mathrm{Z2}$ is the proper version of $\mathrm{Z}$ to report from the transformed code. Since $\mathrm{Z2}$ must be reported (and not $\mathrm{Z1}$ ) it is necessary to distinguish between the $\mathrm{Zs}$ and therefore the $\mathrm{Z2}$ name must be maintained. 
The other reason for not reclaiming names is to allow multiple copies of a variable to be live on different concurrent tasks, thereby enabling the exploitation of parallelism. In this example, A3 cannot share storage with $\mathrm{Al}$, because A1 is simultaneously live on a concurrent process. Similarly A2 cannot share storage with A1 or A3.

The $\mathrm{B} 2$ variable is reclaimable because neither $\mathrm{B} 1$ nor $\mathrm{B} 2$ needs to be available on a concurrent task, nor is B1 live on any concurrent task. The decision to reclaim $B 2$ will result in a change in statement 7 of the parallelized code where B2 becomes $\mathrm{B} 1$, and an accompanying update to the database in the B entry of the AVAIL set associated with statement 7.

This parallelized program with names reclaimed (which is no longer singlevalued) can now be compiled and executed. The programmer, debugging from the viewpoint of the sequential code, places a breakpoint in the sequential code. This breakpoint maps through to the transformed code. When the breakpoint is encountered, a request for a value made by the programmer traps into the runtime interface. This module in turn replaces the variable name requested with the version name associated with the breakpoint position which is stored in the AVAIL data set. The debugger then proceeds to fill the revised request in the ordinary way. In this example, if the programmer places a breakpoint after statement 3 , a request for $\mathrm{X}, \mathrm{T}, \mathrm{A}$ or $\mathrm{Z}$, will be replaced with requests for $\mathrm{X} 1, \mathrm{~T} 1, \mathrm{~A} 2$, or $\mathrm{Z} 1$ respectively and the new requests filled by the debugger. The global renaming and name reclamation processes are presented in greater detail in the following sections.

\section{Global Renaming}

The task of global renaming requires the creation of a new variable name at each variable definition, and also at each program location where divergent execution paths may join. This resolves ambiguity after the join point that may occur in trying to determine which of multiple names (values) should be used. Figure 3 shows this case.
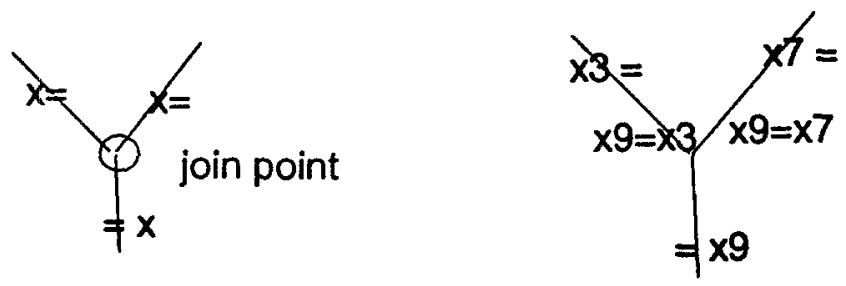

a) before renaming

b) after renaming

Figure 3 - Renaming at join points in program flow.

In addition, global renaming must find blocks of code that may be reentered (loops) and ensure that scalars within such blocks are expanded to vectors. This results in variables with altered types as well as altered names. Figure 4 shows this case. 


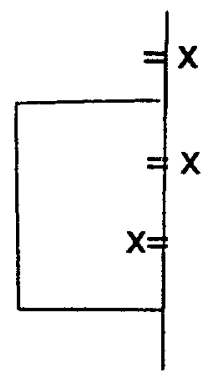

a) before renaming

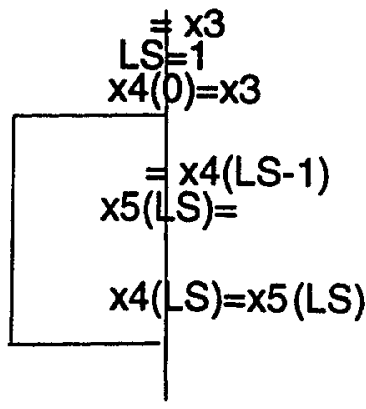

b) after renaming

Figure 4 - Renaming repeated code

In structured code, these join points and loops coincide with structure boundaries. In unstructured code, they are generally discovered by analysis on a Control Flow Graph (CFG). The variety of these approaches has resulted in the development of three distinct global renaming algorithms. The first is an algorithm for structured FORTRAN 77 code ${ }^{[P i s o 91, \text { Pineog3] }}$. This algorithm produces optimal quality code in linear time and recognizes all high-level constructs. It is thus appropriate for use on a large subclass of FORTRAN programs. The second algorithm models unstructured code as a sequence of simple commands in a linear code space, infused with arbitrary GOTO' ${ }^{\text {Primoss] }}$. It is able to assert join points without production of the CFG, and so although it is very general, it still operates in linear time. However this algorithm inserts some unnecessary assignment statements. The most general of the algorithms (and the most expensive) is an extension of the dominance frontier algorithm of Cytron, Ferrante, Rosen, Wegman and Zadeck ${ }^{\text {(crewzil) }}$ which in its original form produces Static Single Assignment (SSA) code from unstructured code in $\mathrm{O}\left(\mathrm{n}^{3}\right)^{\left|\mathrm{Pim}_{\mathrm{m}} \otimes 3\right|}$.

The extension necessary to tailor this algorithm to create single assignment code occurs in the discovery and renaming of loops. Each loop discovered in the CFG is assigned a unique looping subscript (analogous to the LS of Figure 4). Individual statements may belong to any number of loops. Variables defined have subscripts added according to loop membership of the statement. The size of the arrays is determined by the loop bounds. If the bounds of the loop are unknown, vectors are allocated as needed in "chunks" of fixed size. In practice these variables are often reclaimed before execution and many of these allocations do not occur.

Although the examples show only scalar variables, array variables are also renamed using analogous techniques. Any time an array is altered it is renamed, initiating a copy into a new array object. The expansion of array objects thus creates arrays of arrays.

While the renaming of arrays continues to remove all anti and output dependencies, it also has the effect of increasing the number of flow dependencies. These come about because the copying of an array object is dependent on the expression defining the new element as well as the last current array object. The array assignment $A[7]=X$ is renamed as $A 2=\operatorname{copy}[A 1,7, X 1]$. The renamed code explicitly shows the dependency of the statement on both $\mathrm{X} 1$ and $\mathrm{A} 1$. The global renaming stage removes these introduced flow dependencies when it can be 
determined that they are unnecessary. These approaches and a more detailed discussion of global renaming are described in [Pineo93].

\section{Name Reclamation}

After the globally renamed program has been partitioned and parallelized, it is the task of name reclamation to eliminate the unnecessary names. This is accomplished in three steps by first computing the maintenance ranges of the values, then reclaiming the unnecessary names, and finally updating the AVAIL sets to reflect the changed names.

\subsection{Computing Maintenance Ranges}

As seen previously, there are two reasons for maintaining a name: 1) it is still live, 2) it still needs to be available for debugging. This requires the computation of a maintenance range for each value that includes the entire live range of the value and also its Available range. Symbolically,

$$
\mathrm{MR}_{v}=\mathrm{R}_{\mathrm{Av}} \cup \mathrm{R}_{\mathrm{Lv}}
$$

where $R_{A v}$ is the available range of the value computed in the sequential code and mapped into the transformed code, and $R_{\mathrm{Lv}}$ is the live range of the value in the transformed code.

It is straightforward to calculate $R_{A v}$ by standard live range analysis with extensions to include statements up through the value redefinition. This is computed by the global renaming stage and stored in the AVAIL data set. However it is then incumbent upon the name reclamation stage to map these availability ranges into the transformed code. In this stage it is necessary to view both the AVAIL sets and the transformed code to determine when specific variables must be available to serve debugging requests in the transformed program. Discrete locales of availability are combined into one contiguous availability range, since variables are assigned only once and can therefore become available only once.

In the computation of $R_{\mathrm{Lv}}$, it is assumed that the transformed program may be modified for some form of parallel execution. A live range for a value may end on a certain processor, but if the value is also live on a parallel task, it cannot be considered dead until there is a synchronization point between the tasks. Therefore live range analysis in a parallel environment requires an inspection of all subtasks that will be in concurrent execution. If a variable is live in only one subtask P1, then the variable dies when the last use is past. However, if the variable is also live on another task P2, then the variable is not dead until the P1P2 synchronization following the last use. Furthermore, the variable is not completely dead until dead on all subtasks.

To illustrate these computations using the code of Figure 2, the Al variable is available at statements S1-S2, and must be live at S1-S2. However, since $\mathrm{S} 1$ and $\mathrm{S} 2$ are on concurrent tasks the live range is extended to the synchronization point. Therefore $\mathrm{MR}_{\mathrm{A1}}=\mathrm{S} 1-\mathrm{S} 7$. Variable $\mathrm{Z} 1$ has an Avail range of $\mathrm{S} 2-\mathrm{S} 3$ and live range of $\mathrm{S} 2-\mathrm{S} 3$, giving $\mathrm{MR}_{\mathrm{z1}}=\mathrm{S} 2-\mathrm{S} 3$. For variable $\mathrm{Z} 2$ the Avail range, $\mathrm{S} 4-\mathrm{S} 7$, and live range, $\mathrm{S} 4$, cross parallel tasks giving a giving a maintenance range $\mathrm{MR}_{\mathrm{zz}}=\mathrm{S} 1-\mathrm{S} 7$. 
Two variables are said to have overlapping maintenance ranges if they must both be maintained at the same time, as in the case of $\mathrm{Z1}$ and $\mathrm{Z} 2$ above. When the two variables have the same root name, eg., $\mathrm{X} 1$ and $\mathrm{X} 3$, and nonoverlapping ranges, it is always safe to reuse the address. Symbolically,

$$
\begin{aligned}
& \text { if } \mathrm{MR}_{\mathrm{V}} \cap \mathrm{MR}_{\mathrm{V}}=\varnothing \\
& \text { and Root }\left(\mathrm{V}_{1}\right)=\text { Root }\left(\mathrm{V}_{2}\right) \\
& \text { then } \quad \mathrm{V}_{2}=@ \mathrm{~V}_{1} .
\end{aligned}
$$

The availability of a value can be seen as a further use in generating maintenance ranges. If viewed in this way, the maintenance range within a basic block can be simply defined as beginning at the first position of use of the variable and extending to the last.

In name reclamation, maintenance ranges are computed for each variable in each basic block. These "per block" maintenance ranges are used to create summary maintenance information, such that at each statement it is known whether the maintenance of a particular variable is required at any time prior to this statement, or at any time beyond this statement. This information is derived from the Control Flow Graph of the program. Backedges are removed from this graph since the reaching definitions of loop variables are handled by explicit mechanisms in renaming. In addition, irreducible flow graph constructs are resolved by removing edges representing backward branches in the written code. The resulting acyclic CFG is used to determine predecessor and successor blocks. Since there may also be concurrent blocks in the CFG, a block $X$ that is concurrent to a block $\mathrm{Y}$ is considered both a predecessor and successor to $\mathrm{Y}$.

This graph is then used to create three maintenance sets per block. A Maintenance Range, set is computed, which holds a minimum and maximum program location for each variable used or available in Basic Block. The computation of availability makes use of original statement line numbers that have been appended to the statement during renaming. These numbers indicate the original statement locations of lines of program code. After the application of program transformations, these numbers will normally be unordered and, in addition, may contain duplicated or missing numbers. However, these numbers provide crucial mapping information. Each time a statement line number is encountered, the associated AVAIL set is queried and any variable available at this line has its maintenance range updated with the present program location (in the transformed program).

After the $\mathrm{MR}_{\mathrm{i}}$ sets are computed for the blocks, they are used to compute boolean sets, $\mathrm{Pre}_{i}$ and Post $\mathrm{i}_{i}$ for each block. Pre $\mathrm{i}_{i}$ contains a bit for each variable indicating whether the variable has a maintenance range in any predecessor of $\mathrm{BB}_{\mathrm{i}}$ (including concurrent blocks). $\operatorname{Pre}_{\mathrm{i}}$ is calculated from the immediate predecessors of $\mathrm{BB}_{\mathrm{i}}$ by

$$
\begin{aligned}
& \text { Pre }_{1}=\varnothing \\
& \operatorname{Pre}_{i}=U\left(\operatorname{Pre}_{j} \cup M_{j}\right) \quad \text { where a non-zero entry in } M_{j}, m_{j} n_{k} \\
& \mathrm{j} \text { an imm pred } \\
& \text { of } \mathrm{BB}_{1} \text { or concurrent } \\
& \text { defines a true state }
\end{aligned}
$$


Post similarly indicates variables that have maintenance ranges in any successor to $\mathrm{BB}_{i \cdot}$ Post is calculated in inverse program order from immediate successors and concurrent blocks by

$$
\begin{aligned}
& \text { Post }_{\text {wat }}=\varnothing \\
& \text { Post }_{i}=\underset{j \text { an imm succ }}{\cup}\left(\text { Post }_{\mathrm{j}} \cup \quad \mathrm{MR}_{\mathrm{j}}\right. \text { ) } \\
& \text { of } \mathrm{BB}_{\mathrm{i}} \text { or concurrent }
\end{aligned}
$$

\subsection{Reclaiming the Names}

After the maintenance sets have been computed, names can be reclaimed from the code. The injunction against values sharing a variable name when they have overlapping maintenance ranges allows name reclamation to be modelled as a graph coloring problem. The graph consists of vertices $v_{i}$ corresponding to each value generated. There is an edge from $v_{i}$ to $v_{j}$ whenever $v_{i}$ and $v_{j}$ may not share a variable name. Specifically this results when any of the following is true:

1) the variables have different root names,

2) the variables have differing dimensionality, or

3) the variables have intersecting maintenance ranges.

At the beginning of the name reclamation process, this graph contains $\mathbf{n}$ vertices and is colored in $\mathbf{n}$ colors, where $\mathbf{n}$ is the number of variables in the globally renamed program. Name reclamation seeks to recolor this graph, using fewer colors. The reclaimed colors represent names that will not appear in the final executable program.

The graph is traversed starting from any arbitrary node. A color pool is maintained which represents the set of names that have been evaluated and will be retained. This set corresponds to the set of names finally beld by the visited nodes. As the graph is traversed, an attempt is made to recolor each new node encountered with a color already in the color pool. Each candidate color is tried until one is found that has no conflict with the new node, or the list is exhausted. If the node cannot be recolored (the name cannot be reclaimed) then the node's original color is retained and added to the color pool.

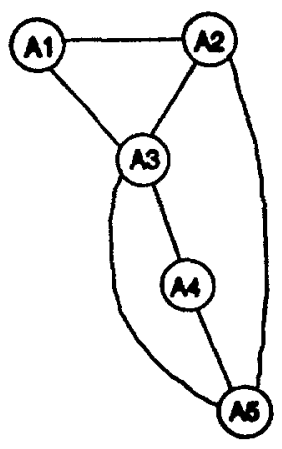

Figure 5 - Name Reclamation by Recoloring 
Figure 5 shows a globally renamed program containing five names, with maintenance range intersections (conflicts) shown as edges. The algorithm starts with an empty color pool and immediately adds A1 to the color set. A2 and A3 are also added because conflicts in the graph do not allow any of these names to share storage. In processing node A4, all colors in the pool (in last-added order) will be tested until one is found that does not conflict with A4. If no such color were found, A4 would be retained. However, in this case, after A3 is rejected, $\mathrm{A} 2$ is selected to replace A4. In the processing of the A5 node, A3 and A2 are rejected but $\mathrm{Al}$ is selected. The resultant graph contains three names.

The algorithm presented does not compute a minimal name space, as the computation of a minimal name space is an NP-complete problem by a trivial polytransformation from graph coloring. Figure 5 shows that extra names may occasionally be allowed by this algorithm. A4 can be reclaimed by choosing to subsume A4 into either A1 or A2. The choice of A2 as described above will allow A5 to be reclaimed as well (subsumed by A1). However, had the A2 and A1 names been encountered in reverse order, causing $A 1$ to be tried first and chosen, the choice of A1 for A4 forces A5 to be unnecessarily retained. The algorithm tries all active names starting with the last retained and the arbitrariness of this ordering allows nonoptimal name choices to be made in transformed programs. In practice extra names occur infrequently because conflict graphs tend to be characterized by many nodes and few edges.

Computing the maximal degree in the graph allows an upper bound to be placed on the number of colors required $=$ maxdegree +1 . In the graph of Figure 5 the maximum degree is four, and the graph is recolored using three colors. To observe that maxdegree+1 represents an upper bound on retained names in name reclamation, consider the recoloring of the ith node where the degree of node $<=$ maxdegree. Assume also that the pool of available colors contains $<=$ maxdegree+1 colors. There are $<=$ maxcolors adjacent to node ${ }_{i}$ If the color pool contains maxdegree+1 colors, then there exists at least one color not represented on nodes adjacent to node $\mathrm{i}$. This color can be chosen for node. If the color pool contains < maxdegree+1 colors, then node,'s color can be retained and added to the pool. After the coloring of node, the color pool still contains $<=$ maxdegreet 1 colors.

In untransformed programs, each new definition kills the range behind it and thus there are no maintenance range intersections. As there are therefore no edges, all names are reclaimed except one (i.e., $X \Rightarrow X 1$ ).

A criticism of coloring algorithms may be that implementation becomes prohibitively expensive because the graphs, involved get quite large. This is especially true for graphs created with single assignment programs. In practice the reclamation algorithm may be implemented without building the graph, using the pre, post and MR sets described above. Collectively they allow the existence of a conflict edge to be efficiently computed.

At each statement the name of a defined variable, V2 may be reclaimed if there exists another variable, V1, previously unreclaimed, such that V1 has the same root name and dimensionality as $\mathrm{V} 2$ and the two variables possess nonintersecting maintenance ranges. This last condition is computed by checking 
that $\mathrm{MR}_{\mathrm{v} 1} \cap \mathrm{MR}_{\mathrm{v} 2}=\varnothing$ within the block, that $\mathrm{V} 2$ has no maintenance range in a predecessor block and that V1 has no maintenance range in a successor block.

If the maintenance ranges are disjoint then the active name replaces the new name and the new name is reclaimed. This also causes maintenance sets for the active variable to be updated. If no active name can be found, the new name is retained and added to the active set.

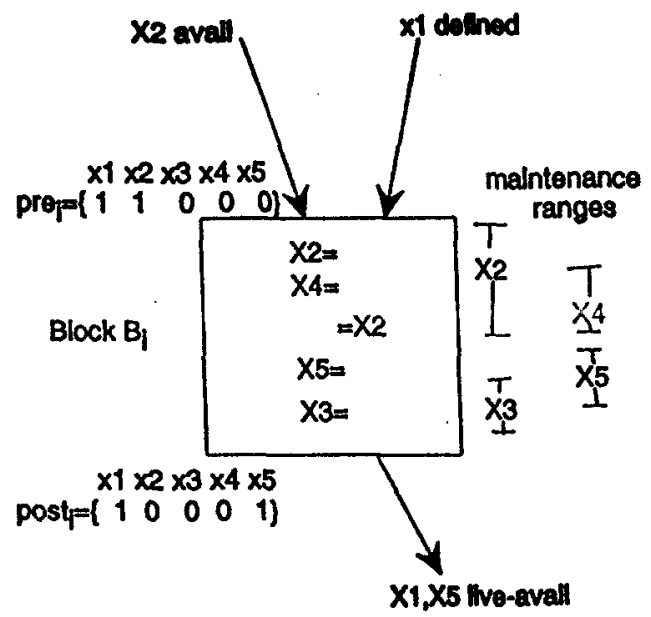

Figure 6 - Name reclamation in a basic block

Figure 6 illustrates the action of name reclamation. The example is simplified by showing only a single root name. The basic block shown is associated with a pre and post set. These indicate that $\mathrm{X} 1$ and $\mathrm{X} 2$ have maintenance ranges prior to $\mathrm{B}$ and that $\mathrm{X} 1$ and $\mathrm{X} 5$ have maintenance ranges after B. Beside the block the (contiguous) maintenance ranges are displayed. Active names that reach the block are $\{\mathrm{X} 1\}$. During the processing of the block, $\mathrm{X} 2$ will not be reclaimed by $\mathrm{X} 1$ because $\mathrm{X} 2$ has a previous maintenance range, and also because $\mathrm{X} 1$ has a later maintenance range. $\mathrm{X} 2$ is then added to the active set. $\mathrm{X} 4$ is also retained because it has a nonempty intersection with $\mathrm{X} 2$ and $\mathrm{X} 1$ has a post maintenance range. X5 will be subsumed by X4 because pre (X5) is false, post (X4) is false and $\mathrm{X} 4$ and $\mathrm{X} 5$ have an empty intersection. This reclamation causes updates to Pre(X4) and Post(X4) such that

$$
\begin{aligned}
& \text { Pre(X4) = Pre(X4) } \mid \text { Pre(X5) } \\
& \text { Post(X4) }=\text { Post(X4) I Post(X5). }
\end{aligned}
$$

The inblock maintenance ranges of X4 and X5 are also merged and information is retained that $\mathrm{X} 5$ is benceforth known as $\mathrm{X} 4$ in the ref-pointer set. Now $\mathrm{X} 3$ cannot be subsumed by $\mathrm{X} 4$ because $\mathrm{X} 4$ has inherited post(X5). However, X3 can be subsumed by $\mathrm{X} 2$, and similar set updates are initiated. The block will finally contain only $\mathrm{X} 2$ and $\mathrm{X} 4$.

Another form of name reclamation occurs within loops. The algorithm will recover the expansion of objects when the loop in question was not chosen for parallelization. In the case of nested loops, each loop is associated with a 
unique looping subscript. Those associated with parallelized loops are retained while the others are reclaimed. The reconstruction of the looping subscript portion of each name is done whenever the name is added to the active set.

At the end of a block's processing, the exiting active set is saved for use by successor blocks. After all blocks are processed, the AVAIL database is updated with the name changes and rewritten for later use by the debugger.

A more detailed view of the name reclamation algorithm is given in the Appendix. The efficiency of this algorithm is bounded by $O$ (plen $x$ |varl) where plen is the length of the transformed program, and Ivarl is the number of variables in the transformed program.

\section{Experimental Results}

Global renaming and name reclamation have been implemented in about 3500 lines of $\mathrm{C}$ code in a system designed for structured FORTRAN 77. The experimental testbed consists of ten FORTRAN programs taken from the EISPACK and FFTPACK collections.

The issues investigated are:

1) Are there significant numbers of non-current variables in parallelized code?

2) What is the storage increase associated with global renaming, and name reclamation?

3) What factors are responsible for unreclaimed names?

4) What percentage of non-current variables remain unreportable using global renaming and name reclamation?

Table 1 shows the storage expansion measured in the testbed programs as they pass through the stages of the system. Storage is measured in words and is recorded for the original program, after global renaming, and after parallelization and name reclamation have been applied. For the purposes of measuring the storage implied by expanded variables in loops, any loop with uncertain bounds is assumed to execute 10 times. $^{2}$. For example, BAKVEC's original 153 memory words grows to 11937 after global renaming, representing an increase of 78 times the original. After parallelization and reclamation, the final storage requirement of BAKVEC is 283 words, or 1.84 times the original.

The degree to which storage is reclaimed varies inversely with the amount or parallelism inherent in the program. Highly parallel programs reclaim fewer names, while programs that undergo no parallelizing transformations have virtually all their introduced names reclaimed. The increases range from 1.1 to 7.3 times. The unusually high enlargement figures associated with $B Q R$ come from a program with deeply nested loops and several large parallelizable loops. The average storage enlargement measured in these programs was over 3900 times after global renaming. Excluding the anomalistic $\mathrm{BQR}$, the average enlargement was still a discouraging 1368 times. However, after name reclamation the average program size was a more reasonable 2.5 times the original.

2 This figure is derived from measurements taken by Knuth ${ }^{[\mathrm{K} m \mathrm{t} 7 \mathrm{]}]}$ who reports the code and execution characteristics of 495 FORTRAN programs. 


\begin{tabular}{|c|c|c|c|c|c|}
\hline program & $\begin{array}{l}\text { (words) } \\
\text { original_stor }\end{array}$ & renamed sto & $\begin{array}{l}\text { times } \\
\text { incr }\end{array}$ & after reclamation & $\begin{array}{l}\text { times } \\
\text { incr }\end{array}$ \\
\hline 1. BAKVEC & 153 & 11,937 & 78 & 283 & 1.84 \\
\hline 2. BALANC & 252 & 33,401 & 129 & 392 & 1.08 \\
\hline 3. BALBAK & 127 & 32,721 & 257 & 257 & 2.02 \\
\hline 4. BANDV & 277 & 735,021 & 2653 & 348 & 1.25 \\
\hline 5. BISECT & 150 & 45,293 & 300 & 190 & 1.27 \\
\hline 6. $\mathrm{BQR}$ & 160 & $4,283,868$ & 26768 & 1170 & 7.31 \\
\hline 7. EZFFTI & 39 & 5,880 & 150 & 171 & 4.38 \\
\hline 8. EZFFTF & 6150 & $23,967,981$ & 3897 & 7060 & 1.15 \\
\hline 9. EZFFTB & 6148 & $28,055,204$ & 4563 & 13848 & 2.25 \\
\hline 10.DCHDC & 60 & 14,560 & 242 & 164 & 2.73 \\
\hline \multirow[t]{2}{*}{ average } & \multicolumn{4}{|c|}{$\begin{array}{c}3903 \\
\text { (without } \mathrm{BQR} \text { 1368) }\end{array}$} & 2.53 \\
\hline & & ble $1-S$ & rage $E$ & Irgement & \\
\hline
\end{tabular}

In these tests the renamed code was parallelized by Parafrase-2, an automatic parallelizing package licensed through the University of Illinois ${ }^{\text {[PFiss }}$ It was noted that the parallelization of globally renamed code was significantly more successful than when the code was not renamed. Many more (and larger loops) were found parallelizable, an effect that was directly attributable to the reduction of data dependencies. More than six times as many program lines were found in parallelized loops using this technique.

program names unreclaimed due to parall $\%$ due to debugging $\%$

1. BAKVEC

2. BALANC $\quad 140$

3. BALBAK $\quad 130$

4. BANDV 71

5. BISECT 40

6. $\mathrm{BQR} \quad 1010$

7. EZFFTI 132

8. EZFFTF 910

9. EZFFTB 7700

10.DCHDC 104

average

$\begin{array}{rrrr}20 & 15 & 110 & 85 \\ 120 & 86 & 20 & 14 \\ 120 & 92 & 10 & 8 \\ 64 & 90 & 7 & 10 \\ 36 & 90 & 4 & 10 \\ 1003 & 99 & 7 & 1 \\ 130 & 98 & 2 & 2 \\ 644 & 71 & 266 & 29 \\ 6506 & 84 & 1194 & 16 \\ 92 & 88 & 12 & 12 \\ & & 82 & 18\end{array}$

Table 2 - Analysis of Unreclaimed Names 
Table 2 shows the analysis of unreclaimed names. Names that are retained because the multiple versions of a variable need to be simultaneously live (as in a parallel loop) were charged to the parallelism column. Conversely, names retained for the purpose of tracking non-current variables were charged to debugging. Where code is reordered aggressively this number of variables charged to debugging can be high (as in the case of BAKVEC), but normally this number is eclipsed by the variables enabling additional parallelism. Over the group of programs, about $82 \%$ of the introduced variables enabled parallelization. The remaining $18 \%$ were required for value tracking non-current variables.

\begin{tabular}{lrrrrr}
\hline Program & Total V & $\begin{array}{c}\text { Non-current VI } \\
\text { untreated code }\end{array}$ & \%Total & \multicolumn{2}{c}{$\begin{array}{c}\text { Unreportable } \\
\text { using technique }\end{array}$} \\
\cline { 3 - 6 } 1. BAKVEC & 288 & 72 & 25 & 1 & 0.3 \\
2. BALANC & 2,499 & 29 & 1 & 0 & 0 \\
3. BALBAK & 432 & 38 & 9 & 0 & 0 \\
4. BANDV & 11,160 & 318 & 3 & 1 & 0.0 \\
5. BISECT & 5,565 & 57 & 1 & 0 & 0 \\
6. BQR & 10,332 & 361 & 3.5 & 0 & 0 \\
7. EZFFTI & 1,960 & 420 & 21 & 15 & 0.8 \\
8. EZFFTF & 12,470 & 1967 & 16 & 0 & 0 \\
9. EZFFTB & 12,335 & 2070 & 17 & 0 & 0 \\
10.DCHDC & 4,225 & 96 & 2 & 0 & 0 \\
& & & & & $0.01 \%$
\end{tabular}

Table 3 - Variable Unreportability at Debug Time

Table 3 shows the measurement of the degree of non-currentness that exists in the parallelized programs. The number of variable instances was computed as the number of program variables times the number of program lines (only lines past the initial declarations and comments were counted). After transformations were applied the number of non-current variable instances (VI) was counted by counting the number of lines at which each variable is non-current (unreportable at debug time) and summing them over the variable set. The percentage of non-current variable instances was computed and averaged. Finally the number of unreportable variable instances using the proposed debugging technique was counted. These are places where, if a variable value were requested during debugging, the software would report the value is unavailable due to transformations applied. The last column shows this figure as a percentage of the total variable instances.

Some interesting results emerge from these tests. First the ballooning of storage after global renaming is quite large. From a low of 78 times expansion to a high of 26,000 times expansion (average 3800 times), clearly globally renamed code is far too unwieldy to be used directly. The large variation in this expansion depends (exponentially) on the depth of nesting in the program and (linearly) on the program length. 
However, name reclamation succeds in reducing the required storage to a manageable increase of 2.5 times the original. Of these unreclaimed names, a large majority are instrumental in increasing the parallelism in the program. The contribution of these $82 \%$ is clearly seen in the improved parallelism figures. The number of lines of code residing in parallelized loops increases an average of 6.4 times.

The experiments show that the existence of non-current variables in parallelized code is a problem. An average of $9.8 \%$ of all variable instances are found in non-current ranges in these programs. This figure was unexpectedly high. Without the debugging technique these would be unreportable at debug time. But using these methods only $0.01 \%$ of variable instances were still unreportable (due to eliminated code or code moved forward).

These results demonstrate the viability of the method. Not only do they show that the rather invasive nature of parallelizing transformations produces a large percentage of non-current variables, but they also seem to indicate that the cost of debugging such code is small. One could argue that only $18 \%$ of the $2.5 \mathrm{x}$ storage increase is due to debugging. Since

$18 \% * 1.5 \mathrm{x}$ (new unreclaimed names) $=.27$

it can be concluded that the storage enlargement cost of debugging transformed code is about one quarter of the original storage.

\section{Conclusions}

As compilers become increasingly autonomous with respect to the restructuring of code, the problem of debugging such transformed code grows in importance. The approach presented in this paper offers significant advantages to the user. It can be used with any transformational package without placing requirements or limitations on the transformations chosen. While the benefits of modular systems design are well-known, this characteristic is particularly useful with parallelizing packages, since the rapid evolution of defined transformations cripples a transformation-dependent approach.

The formation of single assignment code conveys advantages to later stages of code analysis as well. Parallelization is far more successful and all transformations requiring data dependence analysis are simplified. Code partitions are also computed easily. This work suggests that single assignment code captures properties of flow dependence that are so fundamental to the further manipulation of code, especially in a parallel environment, that it is a very appropriate first step to create this form from the input code via global renaming.

Name reclamation makes this a practical and workable approach by removing the unnecessary name allocations. Using this technique, parallelized programs are constructed in modestly expanded spaces, with far more parallelized code. And, most importantly, these programs can be successfully debugged. 


\section{APPENDIX - The Name Reclamation Algorithm}

\section{Algorithm Reclaim Names(P:Procedure)}

1. Compute Maintenance Sets ( $M R_{\text {, }}$ Pre, Post)

2. Process Program Blocks Reclaiming the names

3. Update AVAIL data set with changed names end Reclaim Names

\section{Compute Maintenance Sets}

1. Create Program Dependence Graph - Basic Blocks, with concurrency indicated.

Mark loop heads and delete backedges. Original statements are marked with original statement numbers.

2. Read AVAIL Set associated with original sequential program.

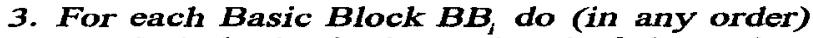

Mark the beginning and end of the maintenancerange $M R$, of each variable used or defined in $B B_{i}$ :

For each program line of transformed program

For each USE or DEF var

if $M R_{Y}(k)$.min undefined then $M R_{Y}(k) . \min =$ program

location

$M R_{Y}(k) \cdot \max =$ program location

For each line number read on input fpointing to original statement location?

for each var $_{k}$ in A VAIL (line number)

if present loc: $<M R(k)$.min or undefined then

MR, $(k)$.min $=$ present location

if present loc $>M R_{,}(k)$.max then

$M R_{Y}(k) \cdot \max =$ present location

4. Calculate Pre, for each $B B_{\text {, }}$ in program order. (Pre is a boolean set indicating which variables have maintenanceranges prior to $\left.B B_{1}\right\}$

$$
\begin{aligned}
& \text { Pre }_{1}=\varnothing \\
& \text { Pre }_{j}=\cup \text { (Prej } \cup M R \mathcal{L} \text { where a non-zero entry in MR.min. } \\
& j \text { an imm pred defines a true state } \\
& \text { of } B B \text {, or concurront }
\end{aligned}
$$

5. Calculate Post for each $B B_{b}$ in inverse program order. Post indicates which variables have maintenance ranges after $\left.B B_{,}\right\}$

$$
\text { Post }_{i}=\cup\left(\text { Post }_{j} \cup \quad\right. \text { MR) }
$$

end Compute Maintenance 


\section{Process Program Blocks}

1. Initialize

Active_set,$=\varnothing \quad$ \{set of active variable names\}

For each variable $k$, ref_pointer $(k)=O$ (ref_pointerpoints to new

2. Rename name if variable is renamed?

For each Basic Block $B B_{i}$ (in program order)

2.1 Update maintenance sets

for all var do

$$
\begin{aligned}
& \text { if } \left.(j=\text { ref pointer }) !=O \quad \text { var }_{j} \text { is new name of } \text { var }_{k}\right\} \\
& \text { then } \operatorname{Pre}_{(j)}=\operatorname{Pre}_{(k)} \text { or } \operatorname{Pre}_{i}(i) \\
& \text { Post }(i)=\text { Posţ }(k) \text { or Post }(j) \\
& M R_{i}(i) \cdot \min =\operatorname{minimum}\left(M R_{i}(k) \cdot \min , M R_{i}(j) \cdot \min \right) \\
& M R_{j}(j) \cdot \max =\operatorname{maximum}\left(M R_{i}(k) \cdot \max , M R_{i}(j) \cdot \max \right)
\end{aligned}
$$
2.2 Compute Active_set $t_{1}=\underset{\text { j-imm prod } \text { BB }_{1}}{\cup}$ Active_set

2.3 Reclaim names in $B B_{i}$

for each USE ( Var $\left._{k}\right)$

if ref_pointer $r_{k}=j(>0)$ then

replace var $_{k}$ with var

for each DEF (vark ), try to reclaim name(until reclaimed or list exhausted):

if ref pointer ${ }_{k}=j(>0)$ (variable already reclaimed\}

then replace var vith $_{v^{\prime}}$ var $_{j}$ else

for each var $_{A}$ in active, set $t_{i}$ with rootname matching var determine whether inaintenance ranges are disjoint: if not Pre $(k)$ (no previous maintenance range Var $_{k}$

and $M R_{1}(k) . \min >=$ present program location and not Post, $(A)$ \{no later maintenancerange for var $_{A}$ \}

and $M R,(A) . m a x<=$ present program location

then \{reclaim var $_{k}$, replace with $\operatorname{var}_{A}$ \}

ref pointer $_{k}=A$

$\operatorname{Post}(A)=$ Post $(k)$

if var $_{k}$ not reclaimed

$M R_{Y}(A) \cdot \max =M R_{Y}(k) \cdot \max$

then \{add var, to active set , $_{j}$ \}

recompute looping subscript, retaining only those associated with parallelized loops

2.4 Save active_set, (to be used by immediate successor blocks ? end Process Blocks

\section{Rewrite AVAIL Sets}

\section{For each line}

For each root name $r$

$$
\begin{aligned}
& \text { let } \operatorname{var}_{k}=A V A I L(i, r) \\
& \text { if ref pointer } r_{k}=j \text { then AVAIL }(i, r)=\operatorname{var}_{j}
\end{aligned}
$$

Rewrite AVAIL set to disk

end Rewrite AVAIL sets 


\section{References}

[BeDa91] M. Benitez and J. Davidson, "Code Generation for Streaming: an Access/Execute Mechanism", 4th ASPLOS Confenence, Santa Cruz, April 1991, pp. 132-141.

[BiNaRo89] L. Bic, M. Nagel, and J. Roy, "Automatic Data/Program Partitioning Using the Single Assignment Principle", Supercomputing 89, pp. 551-556, Aug 1989.

[CoMeRu88] D. Coutant, S. Meloy and M. Ruscetta, "DOC: A Practical Approach to Source-Level Debugging of Globally Optimized Code", SIGPLAN '88 Conf on Prog Lang Design and Impl, Atlanta, GA, June 1988, pp. 125-134.

[CyFe87] R. Cytron and J. Ferrante, "What's in a Name? -or- The Value of Renaming for Parallelism Detection and Storage Allocation", Proceedings of ACM Conference on Parallel Programming. pp 19-27. 1987.

[CFRWZ91] R. Cytron, J. Ferrante, B. Rosen, M. Wegman and K. Zadeck, "Efficiently Computing Static Single Assignment Form and the Control Dependence Graph", ACM Trans on Programming Lang and Systems, October 1991, pp. 451-490.

[Gupt88] R. Gupta, "Debugging Code Reorganized by a Trace Scheduling Compiler," Proceedings of Supercomputing 88 Conference, 1988.

[Henn82] J. Hennessy, "Symbolic Debugging of Optimized Code", ACM Transactions on Programming Languages and Systems, Vol. 4 No. 3, July 1982. pp. 323-344.

[Knut71] D. Knuth, "An Empirical Study of FORTRAN Programs", Software Practice and Experience 1:2, 1971, pp. 105-133.

[PGHLS90] Polychronopoulos, Girkar, Haghighat, Leung, Schouten, "Parafrase-2 User's Newsletter", Center for Supercomputing R\&D, University of Illinois, Urbana Illinois. Fall 1990.

[Pineo93] P.P. Pineo, "The High-level Debugging of Parallelized Code using Code Liberation", Ph.D. Thesis, Department of Computer Science, University of Pittsburgh, April 1993.

[PiSo91] P.P. Pineo and M. L. Soffa, "Debugging Parallelized Code using Code Liberation Techniques", Proceedings of the ACM/ONR Workshop on Parallel and Distributed Debugging, May 20-21, 1991, pp. 102-114.

[PoSo88] L. Pollock and M. L. Soffa, "High-Level Debugging with the Aid of an Incremental Optimizer", Proceedings of the 2lst Hawaii IntI Conference on System Sciences, January 1988.

[Wolf89] M. Wolfe, Optimizing Supercompilers for Supercomputers, MIT Press, 1989.

[Wolf92] M. Wolfe, "Beyond Induction Variables", SIGPLAN '92 conf on Prog Lang Design and Impl, San Francisco, CA, pp. 162-174.

[Zell83] P. Zellweger, "An Interactive High-Level Debugger for Control-Flow Optimized Programs", Proccedings of the ACM Sigsoft/Sigplan Soft. Eng. Symp on High-Level Debugging, March 1983, pp 159-171. 